\title{
Hyaluronate/black phosphorus complexes as a copper chelating agent for Wilson disease treatment
}

\author{
Seong-Jong $\mathrm{Kim}^{\dagger}$, Hye Hyeon $\mathrm{Han}^{\dagger}$ and Sei Kwang Hahn ${ }^{*}$ (])
}

\begin{abstract}
Background: Wilson disease (WD) is a genetic disorder of copper storage, resulting in pathological accumulation of copper in the body. Because symptoms are generally related to the liver, chelating agents capable of capturing excess copper ions after targeted delivery to the liver are highly required for the treatment of WD.

Methods: We developed hyaluronate-diaminohexane/black phosphorus (HA-DAH/BP) complexes for capturing copper ions accumulated in the liver for the treatment of WD.

Results: HA-DAH/BP complexes showed high hepatocyte-specific targeting efficiency, selective copper capturing capacity, excellent biocompatibility, and biodegradability. HA enhanced the stability of BP nanosheets and increased copper binding capacity. In vitro cellular uptake and competitive binding tests verified targeted delivery of HA-DAH/BP complexes to liver cells via HA receptor mediated endocytosis. The cell viability test confirmed the high biocompatibility of HA-DAH/BP complexes.
\end{abstract}

Conclusion: HA-DAH/BP complexes would be an efficient copper chelating agent to remove accumulated copper in the liver for the WD treatment.

Keywords: Black phosphorus nanosheets, Hyaluronate, Chelating agent, Wilson disease

\section{Background}

Wilson disease (WD) is an inborn disorder of copper metabolism and characterized by copper overload in the organs, especially liver and brain [1]. In patients with WD, copper cannot be eliminated properly, causing liver cirrhosis and liver transplantation in severe cases [2]. Accordingly, WD is one of the most challenging diseases in medicine. There are two available treatments to increase copper excretion and reduce copper absorption by using (1) chelators and (2) zinc salts [3]. Combination therapy using zinc salts and chelators leads to clearing

\footnotetext{
* Correspondence: skhanb@postech.ac.kr

†Seong-Jong Kim and Hye Hyeon Han contributed equally to this work and should be considered as co-first authors.

Department of Materials Science and Engineering, Pohang University of Science and Technology (POSTECH), 77 Cheongam-ro, Nam-gu, Pohang, Gyeongbuk 37673, South Korea
}

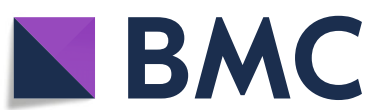

(c) The Author(s). 2021 Open Access This article is licensed under a Creative Commons Attribution 4.0 International License, which permits use, sharing, adaptation, distribution and reproduction in any medium or format, as long as you give appropriate credit to the original author(s) and the source, provide a link to the Creative Commons licence, and indicate if changes were made. The images or other third party material in this article are included in the article's Creative Commons. licence, unless indicated otherwise in a credit line to the material. If material is not included in the article's Creative Commons licence and your intended use is not permitted by statutory regulation or exceeds the permitted use, you will need to obtain permission directly from the copyright holder. To view a copy of this licence, visit http://creativecommons.org/licenses/by/4.0/. The Creative Commons Public Domain Dedication waiver (http://creativecommons.org/publicdomain/zero/1.0/) applies to the data made available in this article, unless otherwise stated in a credit line to the data.

copper overloaded tissues and blocking the pathological accumulation of copper in the organs $[4,5]$. However, the long-term use of the medication is limited because of the safety issues and severe adverse effects [6]. Chelators, such as D-penicillamine and trientine, have been reported to cause adverse events like marrow toxicity, lupus-like syndrome, and anemia [7, 8]. As a suitable chelator, the agent should be able to efficiently capture excess transition-metal ions with excellent biocompatibility and biodegradability. Recently, various nanomaterials have been widely investigated as novel chelators to form nontoxic metal complexes [9]. In addition, nanoparticle-mediated drug delivery efficiently reduced several toxic metals in the body [10]. However, there are few in-depth studies focusing on targeted delivery of chelating agents to the liver for the treatment of WD. 
Here, we developed a hyaluronate-diaminohexane/ black phosphorus (HA-DAH/BP) complex to remove accumulated copper, especially in the liver. BP nanosheets are well known as a biodegradable $2 \mathrm{D}$ material composed of phosphorus atoms [11, 12]. Due to its great biocompatibility, BP nanosheets have been used for biomedical applications including phototherapy, drug delivery, and biocatalysis [13]. Furthermore, phosphorus binds strongly with metal ions, especially $\mathrm{Cu}^{2+}$, making BP nanosheets a robust nanocaptor for copper ions [9]. To prevent the rapid degradation of BP nanosheets from oxidation, BP nanosheets were coated with HA [14]. HA is a natural biodegradable polymer with a high binding affinity toward liver cells [15-18]. After the physicochemical characterization of HA-DAH/BP complexes, we investigated the copper capturing capability, biocompatibility and biodegradability via in vitro liver-specific targeted delivery of HA-DAH/BP complexes for WD treatment.

\section{Methods \\ Materials}

Bulk crystals of black phosphorus (BP) were obtained from Smart-elements (Vienna, Austria). Sodium hyaluronate $(\mathrm{HA}, \mathrm{MW}=10 \mathrm{kDa})$ was acquired from Lifecore Biomedical (Chaska, MN, USA). 1-Methyl-2-pyrrolidinone (NMP), 1,6-diaminohexane (DAH), dimethyl sulfoxide (DMSO), and N-hydroxysulfosuccinimide (NHS) sodium salt were purchased from Sigma Aldrich (St. Louis, MO). 5-Aminofluorescein (FITC) and 1-ethyl-3(3-(dimethylamino)propyl) carbodiimide (EDC) hydrochloride were purchased from Tokyo Chemical Industry (Tokyo, Japan). Cell counting kit-8 (CCK-8) was acquired from Dojindo Molecular Technologies (Kumamoto, Japan). Dulbecco's modified Eagle's medium (DMEM), fetal bovine serum (FBS), and antibiotics were purchased from Gibco (Grand Island, NY). HepG2 cells were purchased from Korean Cell Line Bank (Seoul, Korea).

\section{Preparation of BP nanosheets}

BP nanosheets were prepared by liquid exfoliation technique. In brief, $30 \mathrm{mg}$ of $\mathrm{BP}$ was dispersed in $30 \mathrm{~mL}$ NMP solution with sodium hydroxide. Then, the solution was sonicated for $10 \mathrm{~h}$ (amplifier: 20\%, on/off: $6 \mathrm{~s} /$ $4 \mathrm{~s})$. The non-exfoliated BP nanosheets were removed using centrifugation for $10 \mathrm{~min}$ at $5000 \mathrm{rpm}$ and the supernatant was centrifuged again for $15 \mathrm{~min}$ at 12,500 $\mathrm{rpm}$ to re-disperse BP nanosheets in distilled (DI) water.

\section{Preparation of HA-DAH/BP complexes}

HA-DAH was synthesized by the EDC chemistry between HA (10 kDa, $100 \mathrm{mg})$ and DAH (578 mg, $10 \mathrm{M}$ ratios to HA) in DI water at $\mathrm{pH} 4.8$ for $24 \mathrm{~h}$. Then, HA-
DAH was dialyzed against $15 \%$ ethanol and DI water, respectively $(\mathrm{MWCO}=3500 \mathrm{Da})$. HA-DAH was freezedried for 3 days. After that, BP nanosheets were mixed with HA-DAH at an equal content and stirred for $1 \mathrm{~h}$ to form HA-DAH/BP complexes. The solution was centrifuged for $15 \mathrm{~min}$ at $12,500 \mathrm{rpm}$ to remove excess HA$\mathrm{DAH}$ and obtained HA-DAH/BP complexes were dispersed in DI water.

\section{Characterization of BP nanosheets and HA-DAH/BP complexes}

The prepared BP nanosheets and HA-DAH/BP complexes were analyzed by dynamic light scattering (DLS, Zetasizer Nano ZS90, Malvern Instruments Co., Malvern, UK), UV/vis spectrophotometry (S-3100, Scinco Co., Seoul, Korea), Fourier transform - infrared spectroscopy (FT-IR, Cary 600, Agilent Technologies), and transmission electron microscopy (TEM, JEM-1011, JEOL Co., Akishima, Japan). The physical structure of BP nanosheets and HA-DAH/BP complexes was analyzed by TEM, and the surface modification of BP nanosheets with HA was assessed by DLS and FT-IR.

\section{Stability and biodegradation tests}

BP nanosheets and HA-DAH/BP complexes were suspended in DI water at an equal concentration and then the absorbance spectrum of each solution was measured for 7 days by UV/vis spectrophotometry.

\section{In vitro release test of FITC}

First, HA-FITC conjugates were synthesized by the EDC chemistry between FITC and HA-DAH with a DAH content of $50 \mathrm{~mol} \%$. FITC/BP complexes were prepared by simple mixing of BP nanosheets and FITC, and HAFITC/BP complexes were also prepared by simple mixing of BP nanosheets and HA-FITC conjugates via electrostatic interaction. Solutions were centrifuged for 15 min at $12,500 \mathrm{rpm}$ to remove excess FITC or HA-FITC. After 0, 48, 96, and $144 \mathrm{~h}$, fluorescent intensity of FITC/ $\mathrm{BP}$ and HA-FITC/BP complexes was analyzed using a microplate fluorometer (Fluoroskan ascent FL, Thermo Scientific). The released amount of FITC was calculated from the decrease of the fluorescent intensity.

\section{Determination of $\mathrm{cu}(\mathrm{Cu})$ content}

$\mathrm{CuSO}_{4}$ aqueous solution $(10 \mathrm{~mL}, 40 \mu \mathrm{M})$ was mixed with $\mathrm{BP}$ nanosheets or HA-DAH/BP complexes solution (10 $\mathrm{mL}, 20 \mu \mathrm{g} \mathrm{mL}^{-1}$ ) for $1 \mathrm{~h}$. BP nanosheets and HA-DAH/ $\mathrm{BP}$ complexes were prepared at the desired degradation time point from day 0 to 6 . After centrifugation for 15 min at $12,500 \mathrm{rpm}$, the concentration of metal ions in the supernatants was measured using an atomic absorption spectrometer (TAS-990, Puxi, China). 


\section{Binding capacity of BP nanosheets and HA-DAH/BP} complexes to metal ions

Each metal ion stock solution was prepared from $\mathrm{CaCl}_{2}$, $\mathrm{Mg}\left(\mathrm{NO}_{3}\right)_{2}, \mathrm{ZnCl}_{2}, \mathrm{FeCl}_{3} \cdot 6 \mathrm{H}_{2} \mathrm{O}$ and $\mathrm{CuSO}_{4} \cdot 5 \mathrm{H}_{2} \mathrm{O}$, respectively. BP nanosheets or HA-DAH/BP complexes solution $\left(10 \mathrm{~mL}, 20 \mu \mathrm{g} \mathrm{mL}^{-1}\right)$ was mixed with $10 \mathrm{~mL}$ of the metal ion stock solution for $1 \mathrm{~h}$. After centrifugation for $15 \mathrm{~min}$ at $12,500 \mathrm{rpm}$, the concentration of metal ions in the supernatants was measured using an atomic absorption spectrometer (TAS-990, Puxi, China). The binding capacity was calculated by the following equation as previously reported elsewhere [9]:

$$
\text { Binding capacity }=\frac{\mathrm{C}_{\mathrm{T}}-\mathrm{C}_{\mathrm{s}}}{\mathrm{C}_{\mathrm{T}}} \times 100
$$

where $C_{T}$ represents the total concentration of metal ions in the mixture and $C s$ represents the concentration of metal ions in the supernatant.

\section{Characterization of BP-Cu and HA-DAH/BP-Cu complexes}

The energy dispersive spectroscopy mapping (EDS mapping) of BP, BP-Cu, HA-DAH/BP, HA-DAH/BP-Cu was performed with a field emission - scanning electron microscope (FE-SEM, JSM-7401F, JEOL, Akishima, Japan).

\section{In vitro biocompatibility test}

Liver cancer cells, HepG2, in DMEM were maintained in a humidified $5 \% \mathrm{CO}_{2}$ incubator at $37^{\circ} \mathrm{C}$. HepG2 cells were seeded at a density of $2 \times 10^{4}$ onto 96-well plates and treated with different concentrations of BP nano- (a)

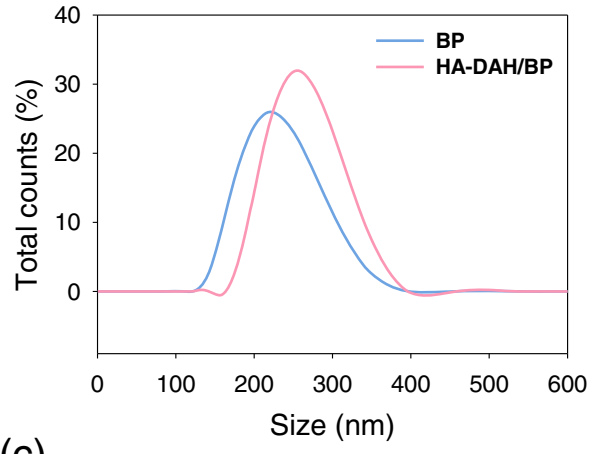

(c)

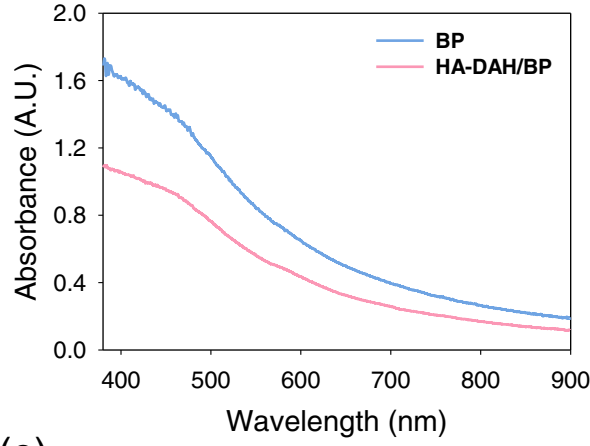

(e)

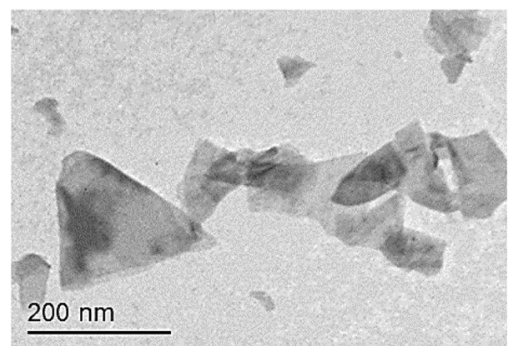

(b)

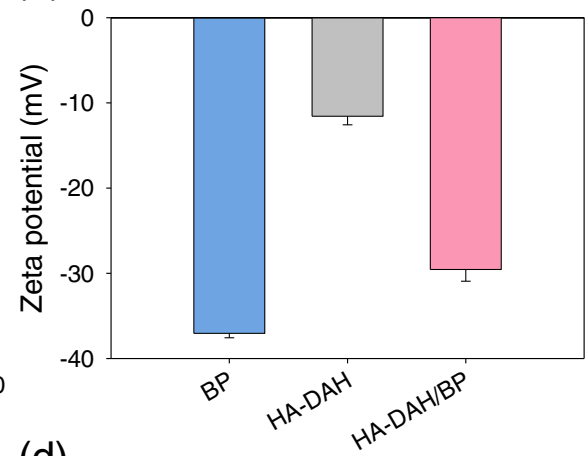

(d)

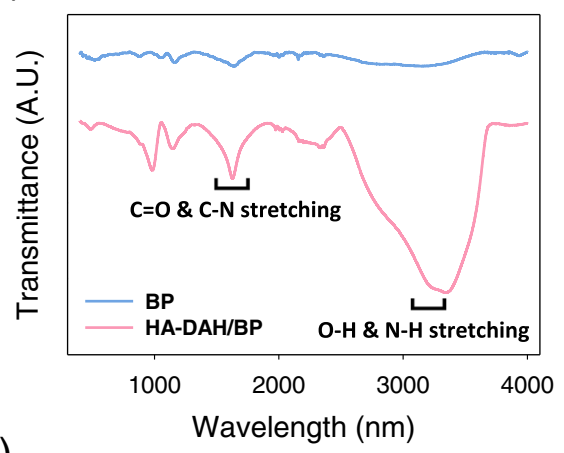

(f)

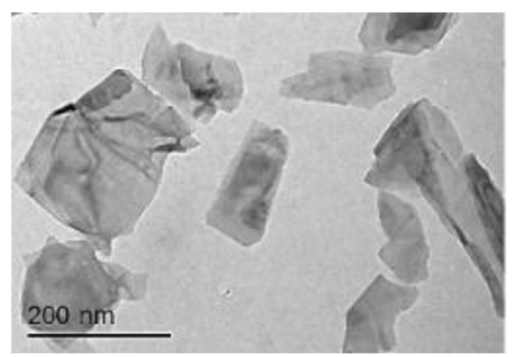

Fig. 1 Characteristics of BP nanosheets and HA-DAH/BP complexes. a Size distribution, b zeta potential, c absorbance spectra and $\mathbf{d}$ FT-IR spectra of BP nanosheets and HA-DAH/BP complexes. TEM images of e BP nanosheets and $\mathbf{f} H \mathrm{~A}-\mathrm{DAH} / \mathrm{BP}$ complexes. Data are expressed as mean \pm SD 
sheets and HA-DAH/BP complexes for $24 \mathrm{~h}$. Serum-free medium and CCK- 8 were added to the cells after washing with PBS. The relative cell viability $(n=4)$ was assessed by the microplate reader (EMax microplate reader, Bucher Biotec AG, Basel, Switzerland).

\section{In vitro cellular uptake}

HepG2 cells were seeded at a density of $1 \times 10^{4}$ cells onto the confocal dish. After incubating for a day, the cells were pre-incubated with excess HA for $2 \mathrm{~h}$ to confirm HA receptor-mediated endocytosis of HA-FITC/BP complexes. FITC/BP or HA-FITC/BP complexes were added, incubated for $2 \mathrm{~h}$, and washed with PBS. The cells were fixed with $4 \%$ paraformaldehyde solution and stained with DAPI. Images were acquired by confocal microscopy (TCS SP5 Ltd., Leica Korea).

\section{Statistical analysis}

Statistical comparison was performed using the software SigmaPlot 10.0 (Systat Software Inc. San Jose, CA). Values for ${ }^{*} P<0.05$ and ${ }^{* * * *} P<0.001$ were considered significant.

\section{Results}

Preparation and characterization of HA-DAH/BP complexes $\mathrm{BP}$ nanosheets were prepared from bulk BP by liquid exfoliation. Then, HA-DAH/BP complexes were synthesized by simple mixing of BP nanosheets with HA-DAH. BP nanosheets and the amino groups of HADAH were physically bound by electrostatic interaction. DLS revealed the size increase from $226.6 \pm 46.6 \mathrm{~nm}$ to $258.8 \pm 42.3 \mathrm{~nm}$ after mixing of BP nanosheets with HADAH (Fig. 1a). The zeta potential of HA-DAH/BP complexes $(-29.6 \pm 1.1 \mathrm{mV})$ was increased from $-37.1 \pm 0.4$ $\mathrm{mV}$ due to amino groups of HA-DAH (Fig. 1b). There was no obvious change in the absorbance spectra of $\mathrm{BP}$ nanosheets after HA coating (Fig. 1c). To confirm the successful synthesis of HA-DAH/BP complexes, FT-IR spectroscopy was performed (Fig. 1d). FT-IR spectrum of HA-DAH/BP complexes showed noticeably increased peaks of $\mathrm{O}-\mathrm{H} \& \mathrm{~N}-\mathrm{H}$ stretching $\left(3000-3500 \mathrm{~cm}^{-1}\right)$ and amide $\mathrm{C}=\mathrm{O} \& \mathrm{C}-\mathrm{N}$ stretching $\left(1600-1700 \mathrm{~cm}^{-1}\right)$. TEM showed that both BP nanosheets and HA-DAH/BP complexes were free-standing with a uniform planar morphology (Fig. 1e and f).

\section{Enhanced stability and biodegradation of HA-DAH/BP complexes}

$\mathrm{BP}$ nanosheets are known to be decomposed into phosphoric acid (PA) in the presence of oxygen [19]. HA can prevent the rapid degradation of BP nanosheets under oxygen circumstance and aggregation with the adsorption of serum proteins $[20,21]$. To assess the effect of

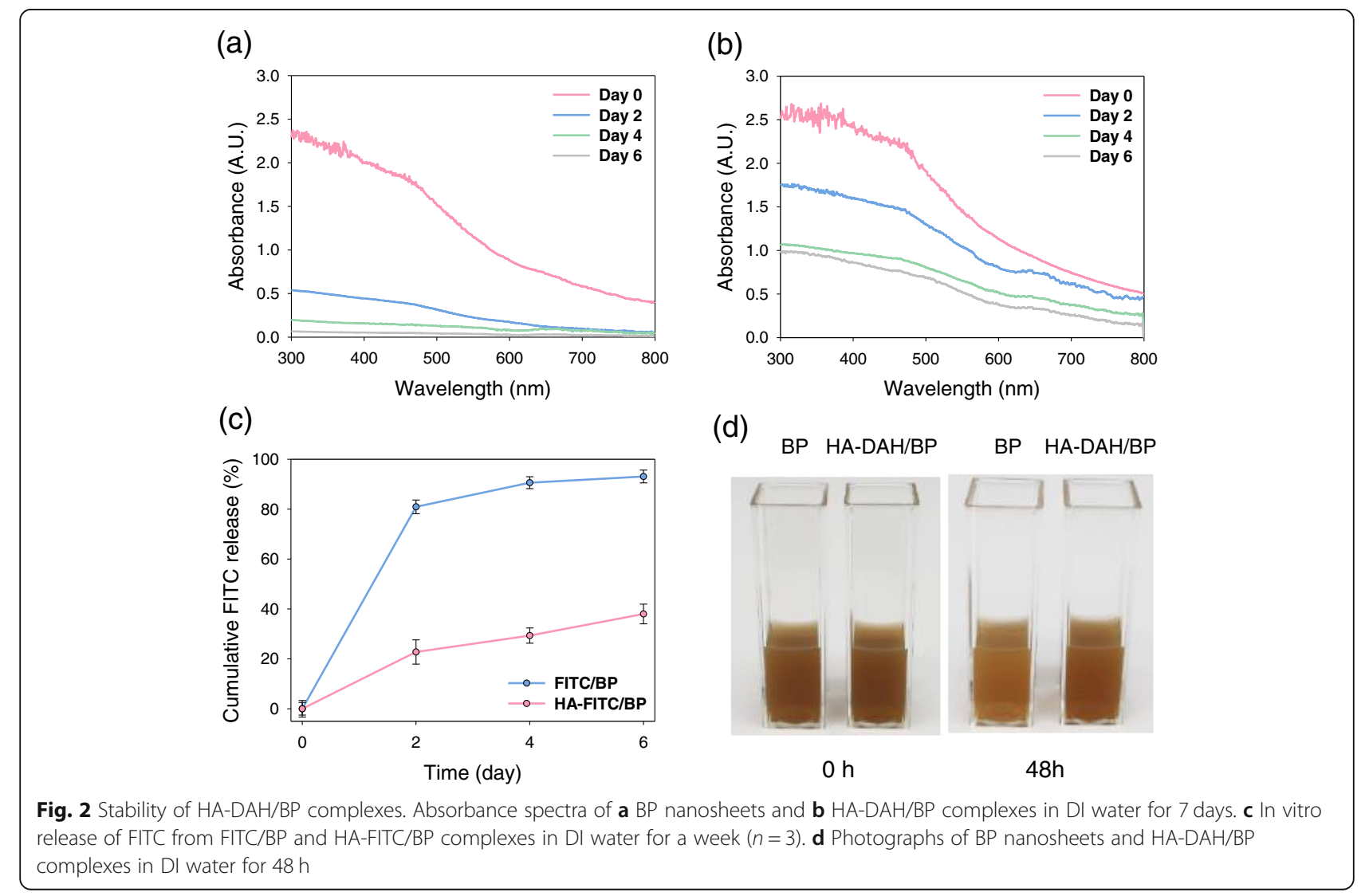


HA on the stability of BP nanosheets, BP nanosheets and HA-DAH/BP complexes at the equal concentration were dispersed in DI water. As shown in Fig. 2a, the absorbance of BP nanosheets was significantly decreased for 7 days and barely remained at day 6 . In contrast, HA-DAH/BP complexes were stable without precipitation and the absorbance spectra was nearly unchanged after 4 days (Fig. 2b). In addition, the enhanced stability of HA-DAH/BP complexes was assessed by the fluorescent intensity of FITC (Fig. 2c). In vitro release of FITC indicated that FITC or HA-FITC was separated from FITC/BP or HA-FITC/BP complexes by degradation. The amount of FITC released from HA-FITC/BP complexes was relatively small with improved stability, and the concentration of FITC was consistent with the degradation rate (Fig. $2 \mathrm{a}$ and $\mathrm{b}$ ). The colour of $\mathrm{BP}$ nanosheets solution became thin after 7 days, compared to that of HA-DAH/BP complexes solution (Fig. 2d). All these results confirmed that HA efficiently protected BP nanosheets from subsequent degradation.

\section{Copper specific capturing of HA-DAH/BP complexes}

The $\mathrm{Cu}^{2+}$ capturing capacity of BP nanosheets and HA$\mathrm{DAH} / \mathrm{BP}$ complexes was investigated by mixing their solutions with $\mathrm{Cu}^{2+}$ solution for $1 \mathrm{~h}$, centrifugation, and dispersion in DI water. The sub-band of $129.3 \mathrm{eV}$ confirms the formation of BP-Cu complexes in the XPS spectrum [9]. TEM images showed that BP nanosheets and HA-DAH/BP complexes maintained their structures after binding with $\mathrm{Cu}^{2+}$ (Fig. 3a). After 7 days, the degraded $\mathrm{BP}$ nanosheets and the degraded HA-DAH/BP complexes interacted with $\mathrm{Cu}^{2+}$. TEM images showed that only HA-DAH/BP complexes still maintained the nanosheets structure binding with $\mathrm{Cu}^{2+}$ (Fig. 3a). The morphology of BP nanosheets disappeared and the spherical shape was obtained only due to copper [22].

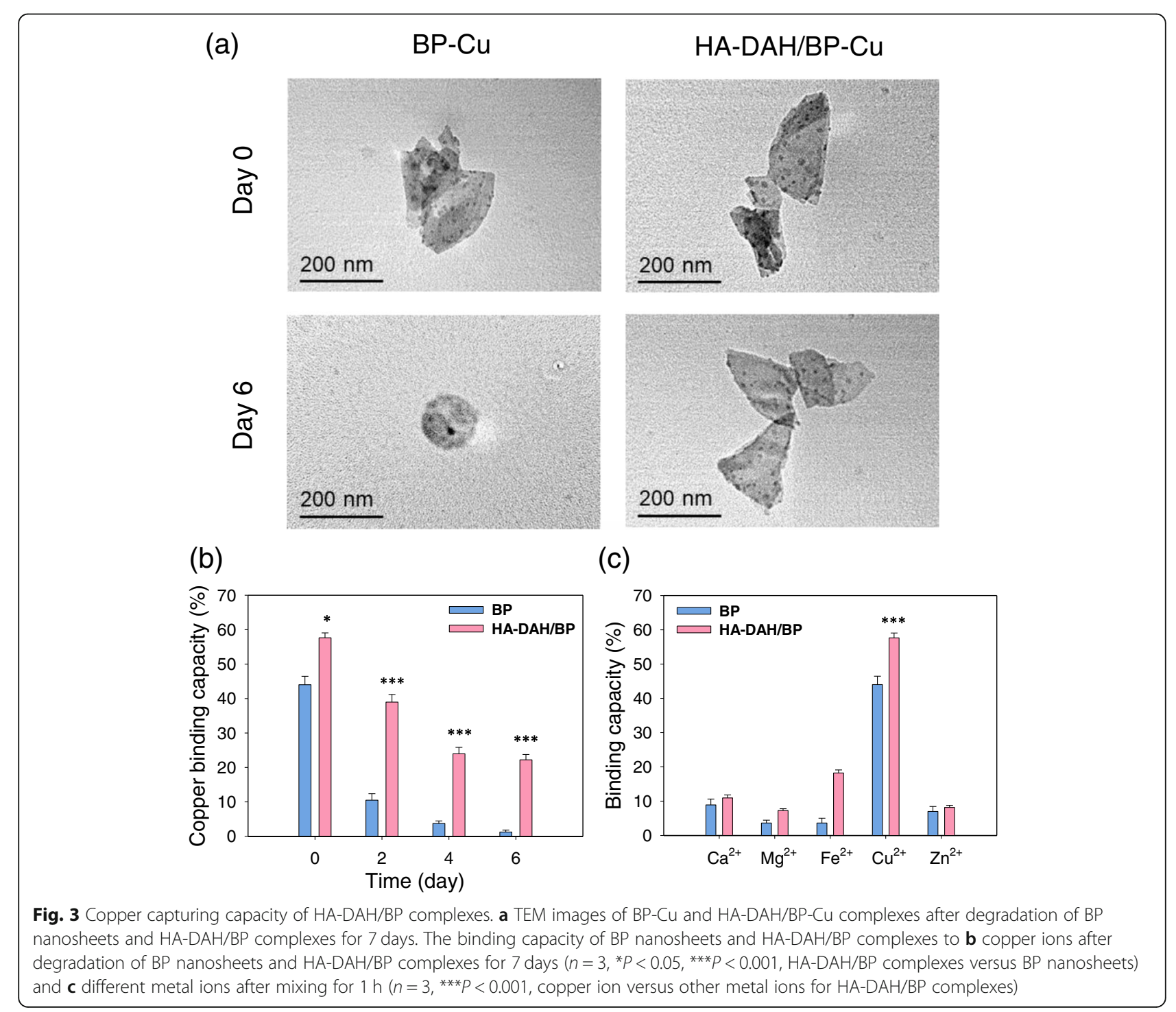


To evaluate the effect of degradation on copper binding capacity, BP nanosheets and HA-DAH/BP complexes were prepared at the desired degradation time point from day 0 to 6 . After mixing the solution of $\mathrm{BP}$ nanosheets or HA-DAH/BP complexes with $\mathrm{Cu}^{2+}$ solution for $1 \mathrm{~h}$, the mixture was centrifuged and the concentration of $\mathrm{Cu}^{2+}$ in supernatants was analyzed with an atomic absorption spectrometer. As shown in Fig. 3b, the $\mathrm{Cu}^{2+}$ capturing capacity of BP nanosheets was dramatically decreased for 7 days. In contrast, the copper binding capacity of HA-DAH/BP complexes was only slightly decreased owing to the HA coating, which was well matched with the degradation rate of HA-DAH/BP complexes in Fig. 2b.

Meanwhile, the copper specific binding of BP nanosheets and HA-DAH/BP complexes was assessed with an atomic absorption spectrometer by comparing the intensity change of each metal ion before and after mixing for $1 \mathrm{~h}$ (Fig. 3c). BP nanosheets and HA-DAH/BP complexes selectively interacted with $\mathrm{Cu}^{2+}$, which indicated the excellent copper capturing specificity. The concentration of other metal ions, such as $\mathrm{Ca}^{2+}, \mathrm{Mg}^{2+}$, and $\mathrm{Zn}^{2+}$ was nearly unchanged. The reason might be that the Gibbs free energy change $(\Delta G)$ of $\mathrm{BP}-\mathrm{Cu}$ or HA-DAH/BP-Cu complexes was much lower than that of BP-metal or HA-DAH/BP-metal complexes [9]. The binding capacity of HA-DAH/BP complexes to $\mathrm{Cu}^{2+}$ and $\mathrm{Fe}^{2+}$ was slightly increased due to the complex formation $[23,24]$.

We next investigated the distribution of copper on BP nanosheets and HA-DAH/BP complexes by the FE-SEM element mapping (Fig. 4). The $\mathrm{C}$ element EDS mapping was only observed in HA-DAH/BP complexes because of HA carbon chains. Copper ions were co-localized with $\mathrm{P}$ elements on the EDS mapping of $\mathrm{BP}-\mathrm{Cu}$ and HA-DAH/BP-Cu complexes. These results successfully demonstrated the high capturing capacity of HA-DAH/ BP complexes to $\mathrm{Cu}^{2+}$.

\section{In vitro cellular uptake and biocompatibility}

The cellular uptake of FITC/BP and HA-FITC/BP complexes into the liver cancer cells, HepG2, was

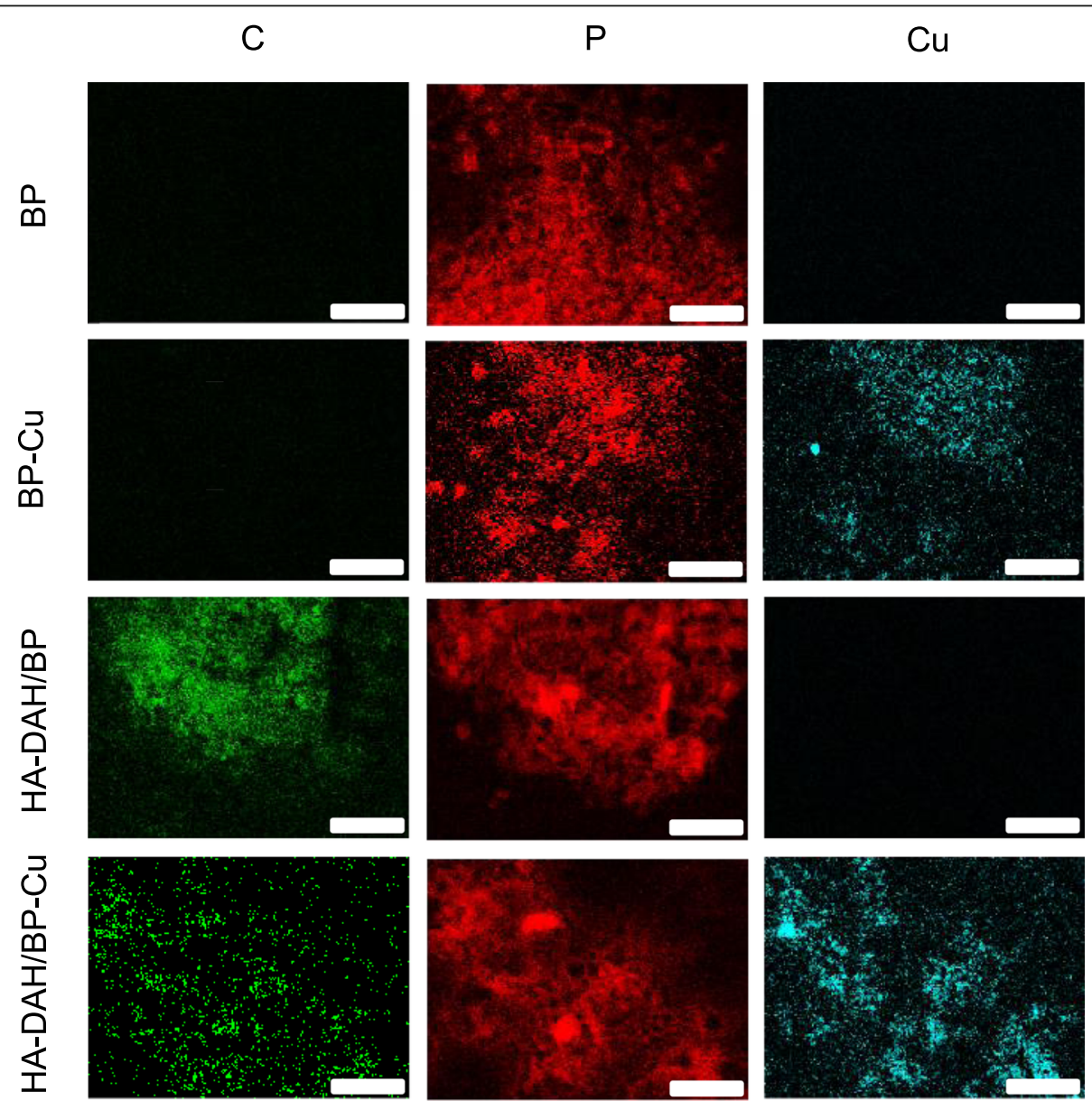

Fig. 4 FE-SEM elemental mapping of $C, P$, and $\mathrm{Cu}$ for BP nanosheets, BP-Cu, HA-DAH/BP, and HA-DAH/BP-Cu complexes (scale bar $=2.5 \mu m$ ) 


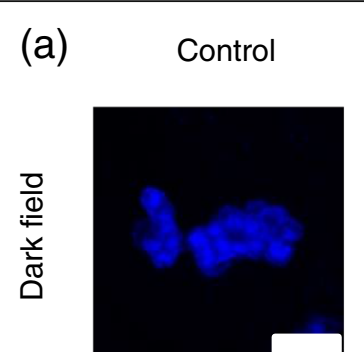

FITC/BP
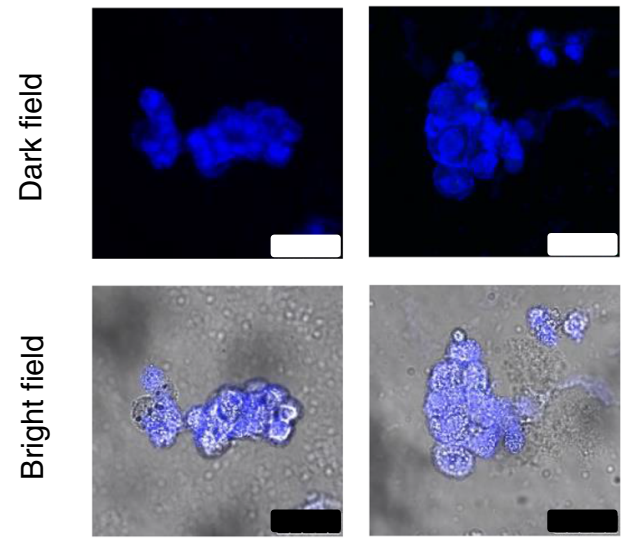

HA-FITC/BP HA-FITC/BP (pre HA)
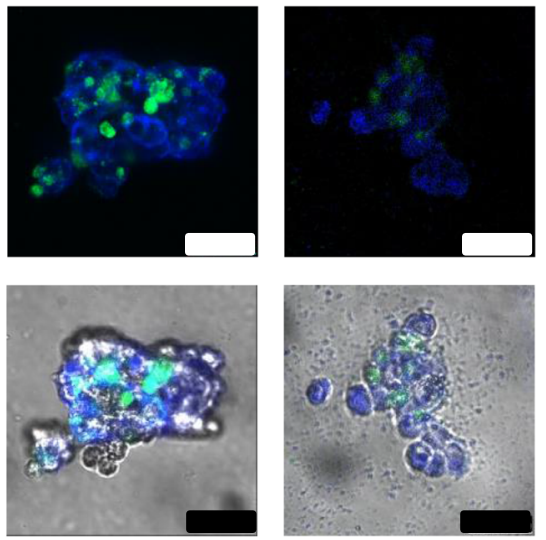

(b)

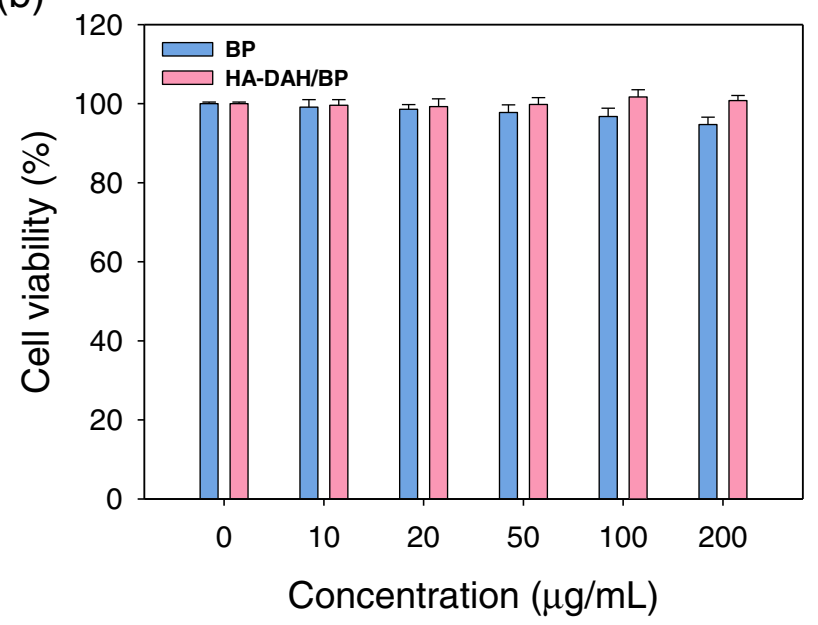

Fig. 5 Cellular uptake and biocompatibility assessment of HA-DAH/BP complexes. a Confocal microscopy of HepG2 cells after treatment with FITC/BP and HA-FITC/BP complexes (scale bar $=50 \mu \mathrm{m}$ ) without and with HA pre-incubation (Blue: DAPI and Green: FITC). b The biocompatibility of BP nanosheets and HA-DAH/BP complexes in HepG2 cells by the CCK-8 assay $(n=4)$. Data are expressed as mean \pm SD

investigated by confocal microscopy. HA-FITC/BP complexes only showed green fluorescence of FITC, indicating the liver cell targeted delivery of HA-DAH/BP complexes (Fig. 5a). HA interacts with liver cells via HA-binding receptors, such as receptor for HAmediated motility (RHAMM) and CD44 [20, 25]. To study the cellular uptake mechanism of HA-FITC/BP complexes, HepG2 cells were pre-treated with excess HA. The fluorescence of HA-FITC/BP complexes was reduced in the cells pre-incubated with $\mathrm{HA}$, which revealed that the uptake of HA-FITC/BP complexes was mediated by HA receptor-mediated endocytosis. The in vitro biocompatibility of BP nanosheets and HADAH/BP complexes was analyzed by CCK-8 assay. As shown in Fig. 5b, the viability of HepG2 cells after treatment with HA-DAH/BP complexes was slightly higher than that with BP nanosheets, which might be ascribed to the biocompatible HA coating $[26,27]$.

\section{Discussion}

This study aimed to protect BP nanosheets from rapid degradation in oxygen circumstances and increase copper binding capacity for WD treatment. Previous studies have reported limitations of BP nanosheets in biomedical applications due to the rapid degradation of BP nanosheets $[13,19]$. As shown in Figs. 2 and 3, BP nanosheets were rapidly decomposed into phosphorus atoms and copper binding capacity also decreased with the degree of decomposition. To overcome this issue, BP nanosheets have been coated with various polymers such as polyethylene glycol (PEG), HA, and poly lactic-coglycolic acid (PLGA) [28, 29]. However, there are no previous studies of the change in the copper binding capacity of BP nanosheets after surface modification. Also, although the liver is the major organ where excess copper accumulates in WD, there are few in-depth studies on delivering chelating agents to the liver for the 


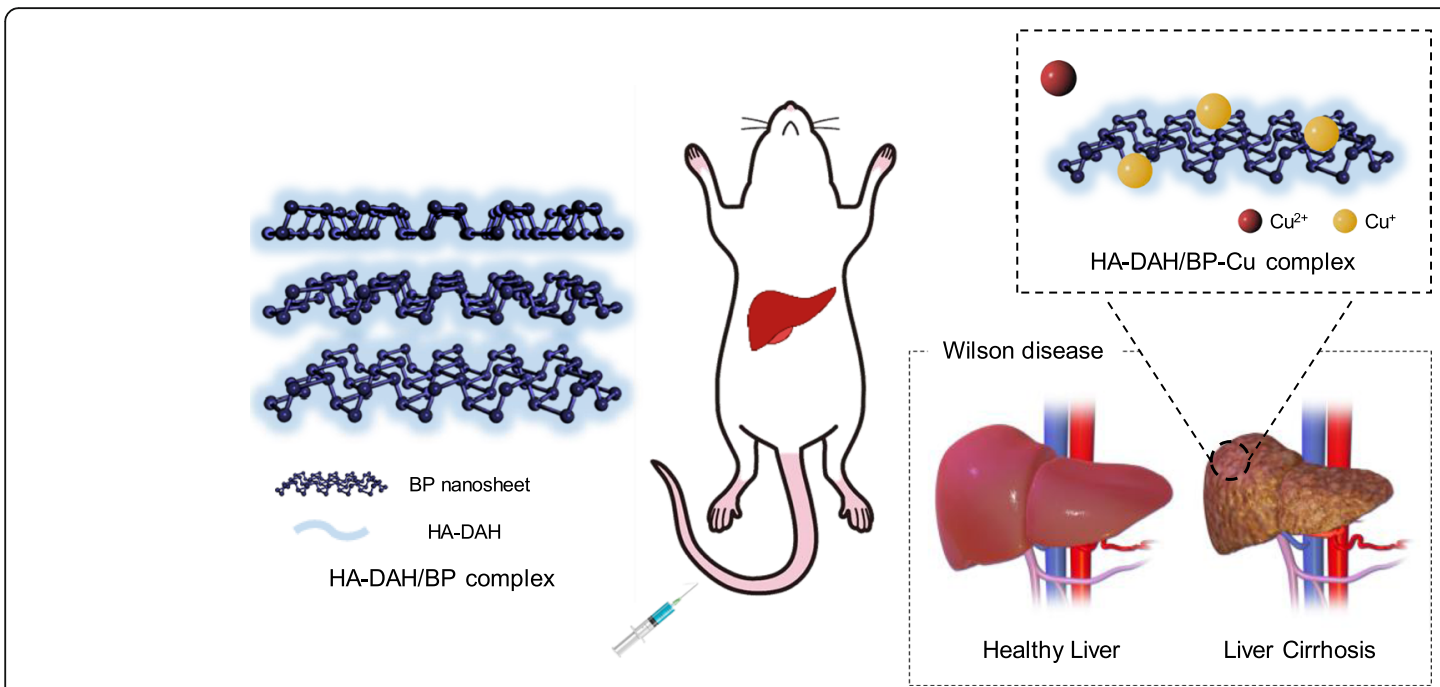

Fig. 6 Schematic illustration of HA-DAH/BP complexes as a copper chelating agent to remove copper ions accumulated in the liver for WD treatment

treatment of WD. In this research, BP nanosheets were modified with HA for improved stability and targeted delivery to the liver. HA has been widely investigated in the biomedical field due to its excellent biocompatibility and targeted delivery to the liver or tumor $[17,18,20]$. Our results demonstrated that HA successfully prevented $\mathrm{BP}$ nanosheets from rapid degradation and maintained the $\mathrm{Cu}^{2+}$ capturing capacity of BP nanosheets after degradation for 7 days (Figs. 2 and 3). The most significant implication was that the copper binding capacity of HA-DAH/BP complexes was higher than that of $\mathrm{BP}$ nanosheets, as shown in Fig. $3 \mathrm{~b}$ and c. The complex formation between $\mathrm{HA}-\mathrm{COO}^{-}$and copper ions increases the copper binding capacity of HA-DAH/BP complexes $[24,30]$. Moreover, the cellular uptake of HA-DAH/BP complexes into the liver cancer cells, HepG2, demonstrated the liver cell targeted delivery of HA-DAH/BP complexes (Fig. 5a). Our results sufficiently showed enhanced stability, copper-specific binding capacity, and hepatic targeted delivery of HA-DAH/BP complexes. This study could be further processed for in vivo animal studies of WD treatment, as schematically shown in Fig. 6.

\section{Conclusion}

We have successfully developed HA-DAH/BP complexes as a copper specific chelating agent for the treatment of WD. HA-DAH/BP complexes could selectively capture $\mathrm{Cu}^{2+}$ among various transition-metal ions, such as $\mathrm{Ca}^{2+}$, $\mathrm{Mg}^{2+}, \mathrm{Fe}^{2+}$, and $\mathrm{Zn}^{2+}$. The copper binding capacity of HA-DAH/BP complexes was significantly higher than that of BP nanosheets. In addition, HA was able to prevent $\mathrm{BP}$ nanosheets from rapid degradation under oxygen environment and maintain the $\mathrm{Cu}^{2+}$ capturing capacity of HA-DAH/BP complexes. With enhanced stability, HA-DAH/BP complexes showed excellent biocompatibility in HepG2 cells. In vitro cellular uptake test with HepG2 cells confirmed the feasibility for targeted delivery of HA-DAH/BP complexes to the liver. Taken together, HA-DAH/BP complexes would be a promising liver targeting and copper chelating agent for WD treatment.

\section{Acknowledgements}

The research was supported by the Basic Science Research Program (2020R1A2C3014070) and the Korea Medical Device Development Fund grant (2020M3E5D8105732).

\section{Authors' contributions}

S.-J.K. and H.H.H. contributed towards the conception and design of this study, acquisition of data, analysis and drafting of the article. H.S.K. conceived of the study, designed the study, coordinated the study and helped draft the manuscript. All authors gave final approval for publication and agree to be held accountable for the work performed therein.

Funding

This work was supported by the grant code: 2020R1A2C3014070, 2020M3E5D8105732.

Availability of data and materials

All data analyzed in this study are included in this published article.

\section{Declarations}

Ethics approval and consent to participate N/A

\section{Consent for publication}

The manuscript has been submitted with the consent of all authors for publication.

Competing interests

The authors declare that they have no competing interests. 
Received: 13 May 2021 Accepted: 31 May 2021

Published online: 16 June 2021

\section{References}

1. Ala A, Walker AP, Ashkan K, Dooley JS, Schilsky ML. Wilson's disease. Lancet. 2007:369(9559):397-408. https://doi.org/10.1016/S0140-6736(07)60196-2.

2. Petrasek J, Jirsa M, Sperl J, Kozak L, Taimr P, Spicak J, et al. Revised King's college score for liver transplantation in adult patients with Wilson's disease. Liver Transpl. 2007;13(1):55-61. https://doi.org/10.1002/It.20920.

3. Roberts EA, Schilsky ML. Diagnosis and treatment of Wilson disease: an update. Hepatology. 2008;47(6):2089-111. https://doi.org/10.1002/hep.22261.

4. Askari FK, Greenson J, Dick RD, Johnson VD, Brewer GJ. Treatment of Wilson's disease with zinc. XVIII Initial treatment of the hepatic decompensation presentation with trientine and zinc. J Lab Clin Med. 2003; 142(6):385-90. https://doi.org/10.1016/S0022-2143(03)00157-4.

5. Santos SEE, Sarles J, Buts JP, Sokal EM. Successful medical treatment of severely decompensated Wilson disease. J Pediatr. 1996;128(2):285-7. https://doi.org/10.1016/50022-3476(96)70412-2.

6. Członkowska A, Litwin T. Wilson disease - currently used anticopper therapy. Hnadb Clin Neurol. 2017;142:181-91. https://doi.org/10.1016/B9780-444-63625-6.00015-X

7. Medici V, Trevisan CP, D'Incà R, Barollo M, Zancan L, Fagiuoli S, et al. Diagnosis and management of Wilson's disease: results of a single center experience. J Clin Gastroenterol. 2006;40(10):936-41. https://doi.org/10.1097/ 01.mcg.0000225670.91722.59

8. Walshe JM. Wilson's disease presenting with features of hepatic dysfunction: a clinical analysis of eighty-seven patients. Q J Med. 1989;70(263):253-63.

9. Chen W, Quyang J, Yi X, Xu Y, Niu C, Zhang W, et al. Black phosphorus Nanosheets as a Neuroprotective Nanomedicine for neurodegenerative disorder. Adv Mater. 2018;30(3):1703458. https://doi.org/10.1002/adma.201 703458 .

10. Liu G, Men P, Perry G, Smith MA. Metal chelators coupled with nanoparticles as potential therapeutic agents for Alzheimer's disease. J Nanoneurosci. 2009;1(1):42-55. https://doi.org/10.1166/jns.2009.005.

11. Kwon H, Seo SW, Kim TG, Lee ES, Lanh PT, Yang S, et al. Ultrathin and flat layer black phosphorus fabricated by reactive oxygen and water rinse. ACS Nano. 2016;10(9):8723-31. https://doi.org/10.1021/acsnano.6b04194.

12. Zhang T, Wan Y, Xie H, Mu Y, Du P, Wang D, et al. Degradation chemistry and stabilization of exfoliated few-layer black phosphorus in water. J Am Chem Soc. 2018;140(24):7561-7. https://doi.org/10.1021/jacs.8b02156.

13. Choi JR, Young KW, Choi JY, Nilghaz A, Lin Y, Xu J, et al. Black phosphorus and its biomedical applications. Theranostics. 2018;8(4):1005-26. https://doi. org/10.7150/thno.22573.

14. Li Y, Feng P, Wang C, Miao W, Huang H. Black phosphorus nanophototherapeutics with enhanced stability and safety for breat cancer treatment. Chem Eng J. 2000;400:125851.

15. Kim H, Jeong H, Han S, Beack S, Hwang BW, Shin M, et al. Hyaluronate and its derivatives for customized biomedical applications. Biomaterials. 2017; 123:155-71. https://doi.org/10.1016/j.biomaterials.2017.01.029.

16. Kim H, Shin M, Han S, Kwon W, Hahn SK. Hyaluronic acid derivatives for translational medicines. Biomacromolecules. 2019;20(8):2889-903. https:// doi.org/10.1021/acs.biomac.9b00564.

17. Lee MY, Yang JA, Jung HS, Beack S, Choi JE, Hur W, et al. Hyaluronic acidgold nanoparticle/interferon a complex for targeted treatment of hepatitis C virus infection. ACS Nano. 2012;6(11):9522-31. https://doi.org/10.1021/nn3 02538y.

18. Oh EJ, Park KT, Kim KS, Kim JS, Yang JA, Kong JH, et al. Target specific and long-acting delivery of protein, peptide, and nucleotide therapeutics using hyaluronic acid derivatives. J Control Release. 2010;141(1):2-12. https://doi. org/10.1016/j.jconrel.2009.09.010.

19. Zhou Q, Chen Q, Tong Y, Wang J. Light-induced ambient degradation of few layer black phosphorus: mechanism and protection. Angew Chem Int Ed. 2016:55(38):11437-41. https://doi.org/10.1002/anie.201605168.

20. Mattheolabakis G, Milane L, Singh A, Amiji MM. Hyaluronic acid targeting of CD44 for cancer therapy: from receptor biology to nanomedicine. J Drug Target. 2015;23(7-8):605-18. https://doi.org/10.3109/1061186X.2015.1052072.

21. Pinto AM, Moreira JA, Magalhaes FD, Goncalves IC. Polymer surface adsorption as a strategy to improve the biocompatibility of graphene nanoplatelets. Colloids Surf B. 2016;146:818-24. https://doi.org/10.1016/j. colsurfb.2016.07.031
22. Cuevas R, Durán N, Diez MC, Tortella GR, Rubilar O. Extracellular biosynthesis of copper and copper oxide nanoparticles by Stereum hirsutum, a native white-rot fungus from Chilean forests. J Nanomater. 2015;10:1-7.

23. Balogh GT, Illés J, Sézkely Z, Forrai E, Gere A. Effect of different metal ions on the oxidative damage and antioxidant capacity of hyaluronic acid. Arch Biochem Biophys. 2003;410(1):76-82. https://doi.org/10.1016/S0003-9861(02 )00661-6.

24. Lapčik L Jr, Dammer C, Valdo M. Hyaluronic acid-copper (II) complexes: spectroscopic characterization. Colloid Polym Sci. 1992;270(10):1049-52. https://doi.org/10.1007/BF00655976.

25. He X, Nie N, Wang K, Tan W, Wu X, Zhang P. In vivo study of biodistribution and urinary excretion of surface-modified silica nanoparticles. Anal Chem. 2008;80(24):9597-603. https://doi.org/10.1021/ac801882g.

26. Kim KS, Kim YS, Bao K, Wada H, Choi HS, Hahn SK. Bioimaging of Botulinum toxin and hyaluronate hydrogels using Zwitterionic near-infrared Fluorophores. Biomater Res. 2017;21(1):15. https://doi.org/10.1186/s40824-01 7-0102-x.

27. Yeom JS, Hwang BW, Yang DJ, Shin HI, Hahn SK. Effect of Osteoconductive hyaluronate hydrogels on Calvarial bone regeneration. Biomater Res. 2014: 18(1):8. https://doi.org/10.1186/2055-7124-18-8.

28. Tao W, Zhu X, Yu X, Zeng X, Xiao Q, Zhang X, et al. Black phosphorus Nanosheets as a robust delivery platform for Cancer Theranostics. Adv Mater. 2017;29(1):1603276. https://doi.org/10.1002/adma.201603276.

29. Zeng G, Chen Y. Surface modification of black phosphorus-based nanomaterials in biomedical applications: strategies and recent advances. Acta Biomater. 2020:118:1-17. https://doi.org/10.1016/j.actbio.2020.10.004.

30. Salehi E, Daraei $P$, Shamsabadi AA. A review on chitosan-based adsorptive membranes. Carbohydr Polym. 2016;152:419-32. https://doi.org/10.1016/j.ca rbpol.2016.07.033.

\section{Publisher's Note}

Springer Nature remains neutral with regard to jurisdictional claims in published maps and institutional affiliations.

\section{Ready to submit your research? Choose BMC and benefit from:}

- fast, convenient online submission

- thorough peer review by experienced researchers in your field

- rapid publication on acceptance

- support for research data, including large and complex data types

- gold Open Access which fosters wider collaboration and increased citations

- maximum visibility for your research: over $100 \mathrm{M}$ website views per year

At BMC, research is always in progress.

Learn more biomedcentral.com/submissions 